\title{
Barriers and facilitators to adoption of and adherence to a Mediterranean style diet in adults: a systematic review of observational and qualitative studies
}

\author{
F. Tsofliou ${ }^{1}$, D. Vlachos ${ }^{1}$ and K.M. Appleton ${ }^{2}$ \\ ${ }^{1}$ Department of Rehabilitation \& Sport Sciences, Faculty of Health \& Social Sciences, Bournemouth University, \\ Bournemouth, Dorset, UK and \\ ${ }^{2}$ Research Centre for Behaviour Change, Department of Psychology, Bournemouth University, Poole, UK
}

Consumption and adherence to Mediterranean diet (MedDiet) has been linked to health benefits such as reduction of overall mortality, reduction in cardiovascular disease risk $^{(1)}$, and some new emerging evidence support a role of MedDiet in mood improvement $^{(2)}$. To promote the implementation of MedDiet efficiently, it is imperative to understand people's views about the difficulties in relation to changing eating behaviour towards a Mediterranean style diet. The present study aimed to conduct a systematic literature review and critically evaluate the current published literature in order to provide an evidence-based insight into the factors that influence adoption and adherence to MedDiet in adults who live inside and outside the Mediterranean region.

The databases Medline, CINAHL \& EBSCO were searched from December 2019 to December 2020 to retrieve relevant studies of adults' perceptions and experiences relevant to following a Mediterranean style diet. Observational studies and qualitative studies were included if they reported on factors that influence adoption or adherence of a Mediterranean style diet in adults 18 years and older or dietary interventions that included food patterns similar to a MedDiet pattern. Studies were excluded if they focused on children or if they included only single components of MedDiet (i.e. "fruit and vegetables" as a single component). The risk of bias was assessed via the STROBE Statement for Observational Studies in Nutritional Epidemiology and with the Critical Appraisal Skills Programme (CASP) tool for qualitative studies.

Of 3,973 retrieved articles, 12 studies fulfilled our inclusion criteria and were included in the review. Of the 12 included studies, four were observational and eight were qualitative studies. Two studies were related to adoption of MedDiet and ten studies were related to adherence to MedDiet. Sample sizes ranged from 11 participants up to 2,000 participants for the qualitative studies and from 43 up to 36,032 participants for the observational studies. Four studies were conducted in United Kingdom and one each in Greece, Netherlands, Italy, Australia, Northern Europe, Norway, Spain and USA. Two studies included females only. Eight influencing factors were identified and categorized into themes: financial restraints, cognitive factors (related to diet knowledge/awareness), sociocultural background (eating habits and traditions), motivational factors (lack of willpower and time pressures), health and lifestyle factors, accessibility \& availability to resources, hedonic factors and demographic factors (age and gender). All these factors, except for motivational and cognitive aspects, were found to influence both negatively and positively the adoption/adherence to MedDiet.

The present findings can expand our understanding of potential facilitating factors and barriers to following a MedDiet. The reported barriers and facilitators to adoption and adherence to MedDiet could be considered in the development of nutrition interventions to promote Mediterranean style diet inside and outside the Mediterranean region.

\section{References}

1. Sofi F, Macchi C, Abbate R, et al. (2014) Public Health Nutr 17(12), 2769-2782.

2. Opie RS, O’Neil A, Jacka FN, et al. (2018) Nutr Neurosci 21(7), 87-501. 